\title{
Chapter 4 \\ Development of Nondestructive Assay of Fuel Debris of Fukushima Daiichi NPP (2): Numerical Validation for the Application of a Self-Indication Method
}

\author{
Tadafumi Sano, Jun-ichi Hori, Yoshiyuki Takahashi, Hironobu Unesaki, \\ and Ken Nakajima
}

\begin{abstract}
To perform decommissioning of the Fukushima Daiichi NPP safely, it is very important to measure the components of the fuel debris. Therefore, a new nondestructive assay to identify and quantify the target nuclide in fuel debris using a pulsed-neutron source is under development in Kyoto University Research Reactor Institute.

We use the self-indication method for the nondestructive assay. This method is a neutron transmission method. The neutron transmission method is focused on resonance reactions (i.e., capture, fission) at the target nuclide. In the self-indication method, the transmitted neutrons from the sample are injected into an indicator. The indicator consists of a high-purity target nuclide. The transmitted neutrons are obtained by the time-of-flight (TOF) technique via resonance reactions in the indicator. The self-indication method has a high signal-to-noise $(\mathrm{S} / \mathrm{N})$ ratio compared to the conventional method.

In this study, numerical validation for the self-indication method to identify and quantify nuclides in a BWR-MOX pellet is described. The burn-up of the MOX pellet is $0 \mathrm{GWd} / \mathrm{t}, 10 \mathrm{GWd} / \mathrm{t}, 20 \mathrm{GWd} / \mathrm{t}, 30 \mathrm{GWd} / \mathrm{t}, 40 \mathrm{GWd} / \mathrm{t}$, and $50 \mathrm{GWd} / \mathrm{t}$. The 12-m measurement line in KUR-LINAC is simulated as a calculational geometry. Numerical calculations are carried out by continuous-energy Monte-Carlo code MVP2 with JENDL-4.0 as the nuclear data library. The burn-up calculations of the BWR-MOX pellet are performed by the deterministic neutronics code SARC 2006 with JENDL-4.0.

Numerical validation for application of the self-indication method is carried out. From the results, it is noted that the self-indication method has a good $\mathrm{S} / \mathrm{N}$ ratio compared to the neutron transmission method for quantifying the amount of target nuclides in the fuel debris.
\end{abstract}

T. Sano $(\bowtie) \bullet J$. Hori $\bullet$ Y. Takahashi $\bullet$ H. Unesaki $\bullet$ K. Nakajima

Kyoto University Research Reactor Institute, 1010, Asashiro-nishi-2, Kumatori-cho,

Sennan-gun, Osaka, Japan

e-mail: t-sano@rri.kyoto-u.ac.jp 
Keywords Burn-up $\bullet$ KUR-LINAC $\bullet$ MOX pellet $\bullet$ Nondestructive assay $\bullet$ Numerical validation $\bullet$ Resonance $\bullet$ Self-indication method

\subsection{Introduction}

To perform decommissioning of the Fukushima Daiichi NPP safely, it is very important to measure the components of the fuel debris. Therefore, a new nondestructive assay to identify and quantify a target nuclide in the fuel debris using a pulsed-neutron source is under development in Kyoto University Research Reactor Institute.

We use the self-indication method for the nondestructive assay. This method is a neutron transmission method. The neutron transmission method is focused on resonance reactions (i.e., capture, fission) at the target nuclide. In the conventional neutron transmission method, a sample is irradiated by a pulsed-neutron beam and the energy distribution of transmitted neutrons from the sample is measured by the time-of-flight technique. Then, the target nuclide in the sample is identified and quantified by using the transmitted neutrons in the resonance energy region. This is a remarkably effective method to identify and quantify the target nuclide. However, if the energy spectrum of the transmitted neutron has many dips caused by resonance reactions of other nuclides, it is difficult to identify and quantify the target nuclide in the sample.

In the self-indication method, the transmitted neutrons from the sample are injected into an indicator, which consists of a high-purity target nuclide. The transmitted neutrons are obtained via resonance reactions in the indicator. The self-indication method has a high signal-to-noise $(\mathrm{S} / \mathrm{N})$ ratio compared to the conventional method.

In this chapter, numerical validation for application of the self-indication method is carried out. A calculational model and conditions are shown in Sect. 4.2 and the numerical results are shown in Sect. 4.3. From these results, some conclusions are drawn in Sect. 4.4.

\subsection{Calculational Model and Condition}

In this chapter, applicability of the self-indication method to identify and quantify nuclides in a BWR-MOX pellet is evaluated. The burnup of the MOX pellet is $0 \mathrm{GWd} / \mathrm{t}, 20 \mathrm{GWd} / \mathrm{t}$, and $30 \mathrm{GWd} / \mathrm{t}$. A plutonium vector in the fresh MOX pellet is employed as the OECD/NEA BWR MOX benchmark $(\mathrm{Pu} 4)\left({ }^{235} \mathrm{U}, 0.2 \mathrm{w} / \mathrm{o}\right.$; total $\mathrm{Pu}, 6.71 \mathrm{w} / \mathrm{o} ;{ }^{238} \mathrm{Pu}, 2.2 \% ;{ }^{239} \mathrm{Pu}, 46.2 \% ;{ }^{240} \mathrm{Pu}, 29.4 \% ;{ }^{241} \mathrm{Pu}, 8.8 \%$ ) [1]. The burn-up calculations of the BWR-MOX pellet are carried out by using deterministic neutronics code SARC 2006 [2] with JENDL-4.0 [3]. The numerical validations are performed by using the MVP2.0 [4] with the JENDL-4.0. The MVP2.0 is a 


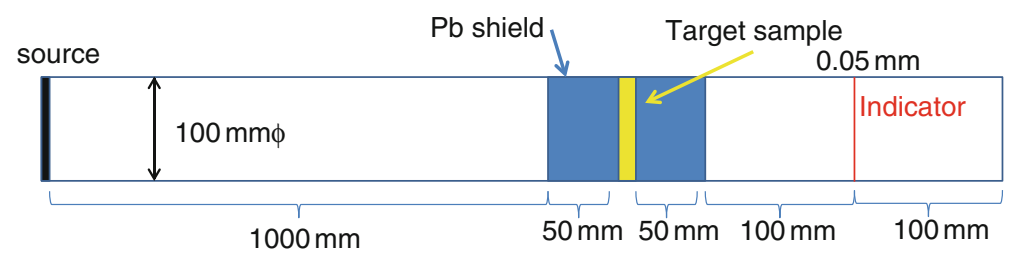

Fig. 4.1 Calculational geometry of 12-m measurement line in KUR-LINAC

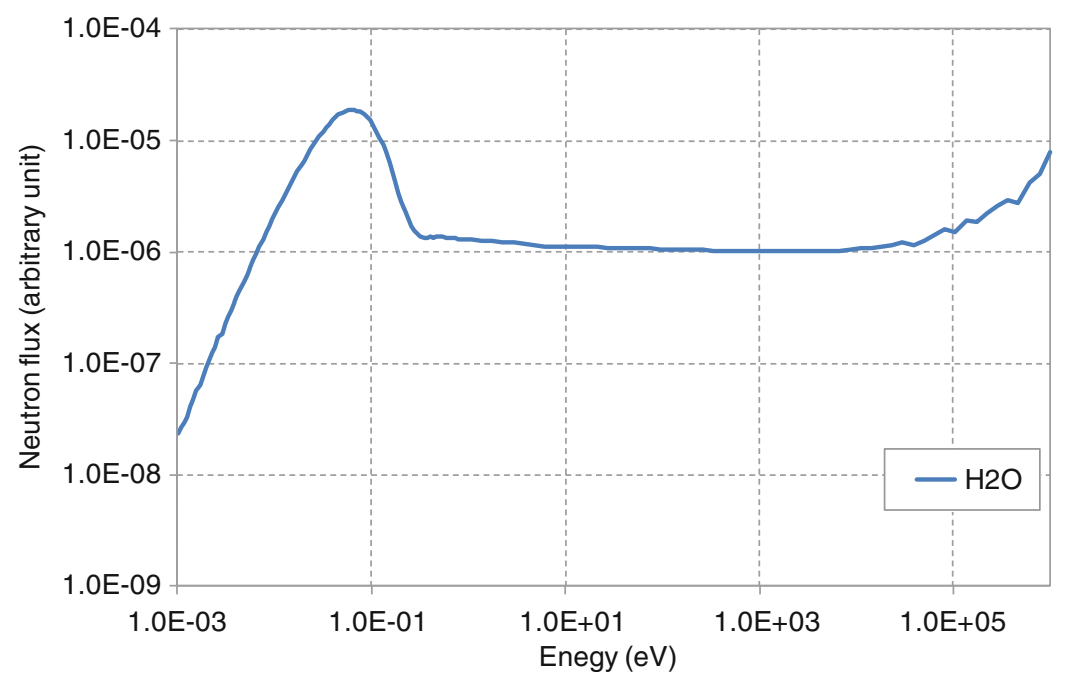

Fig. 4.2 Neutron spectrum in a Ta target of KUR-LINAC

continuous-energy Monte Carlo code developed by the Japan Atomic Energy Agency.

The 12-m measurement line in the KUR-LINAC is simulated as a calculational geometry shown in Fig. 4.1. Figure 4.2 shows a neutron spectrum in a tantalum target that is a neutron source of the KUR-LINAC. The spectrum is calculated by MVP2.0. Using the spectrum as the surface source, the validation is carried out.

\subsection{Numerical Results and Discussion}

The numerical validation for application of the self-indication method is discussed in this section. In the experiment, the transmitted neutron spectrum from the sample is measured via resonance reactions in the indicator whereas the reaction rates in the indicator are shown by numerical calculation. If the energy boundaries are made to have a finer division, the numerical result of the resonance reaction will have the same peak with dips as the measured data. 


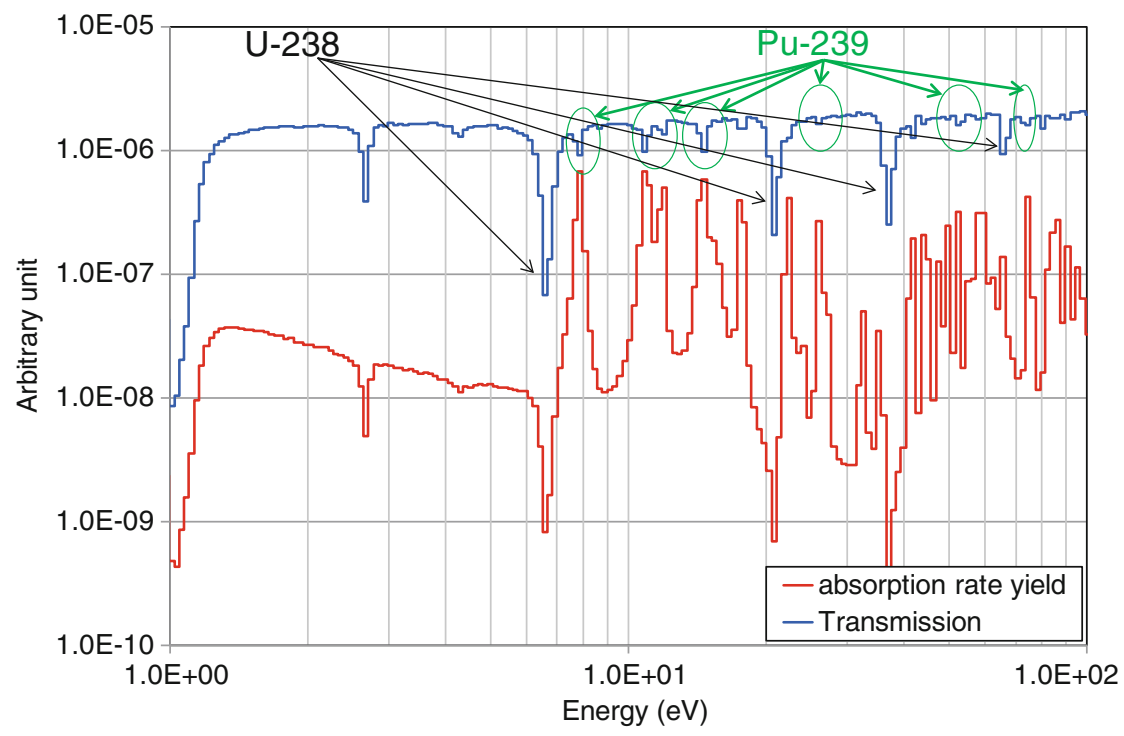

Fig. 4.3 Pu-239 absorption yield in an indicator (sample, $0 \mathrm{GWd} / \mathrm{t}$ )

Figure 4.3 shows the ${ }^{239} \mathrm{Pu}$ absorption rate yield by the present method (red) and the transmitted neutron spectrum by the conventional method (blue). The sample is the fresh (no burn-up) MOX pellet. Using the present method, one can easily obtain resonance absorption by ${ }^{239} \mathrm{Pu}$. On the other hand, the transmitted neutron spectrum has many dips caused by resonance reaction of the other nuclides. Thus, if the sample is a burn-up pellet, it is difficult to quantify and identify by using the conventional method.

A numerical result to identify ${ }^{129} \mathrm{I}$ in the MOX pellet is described. The burn-up of the MOX pellet is $20 \mathrm{GWd} / \mathrm{t} .{ }^{129} \mathrm{I}$ has only four resonances in the energy region of 0.1-100 eV: the resonance peaks are 41, 73, 75, and $97 \mathrm{eV}$. The transmitted neutrons are easily obtained via ${ }^{129}$ I resonance absorption reactions in the indicator by the present method (Fig. 4.4).

Using the self-indication method, one cannot prepare a pure indicator to identify and quantify a target nuclide in a sample. Therefore, it is necessary to validate the application of the present method using an impure indicator. Figure 4.5 shows the numerical result of ${ }^{239} \mathrm{Pu}$ fission yield in the indicator, which has impure plutonium. The sample is a fresh MOX pellet, and the plutonium vector in the indicator is ${ }^{239} \mathrm{Pu}=98.57 \mathrm{w} / \mathrm{o},{ }^{239} \mathrm{Pu}=1.38 \mathrm{w} / \mathrm{o}$, and ${ }^{240} \mathrm{Pu}=0.05$ w/o. In Fig. 4.5, the red line is a pure ${ }^{239} \mathrm{Pu}$ indicator, and the blue line shows that an indicator employed impure plutonium. Even in this case, as well as the result of using the pure ${ }^{239} \mathrm{Pu}$ as the resonance absorption in indicator is observed, it is shown to quantify and identify ${ }^{239} \mathrm{Pu}$ in the sample. 


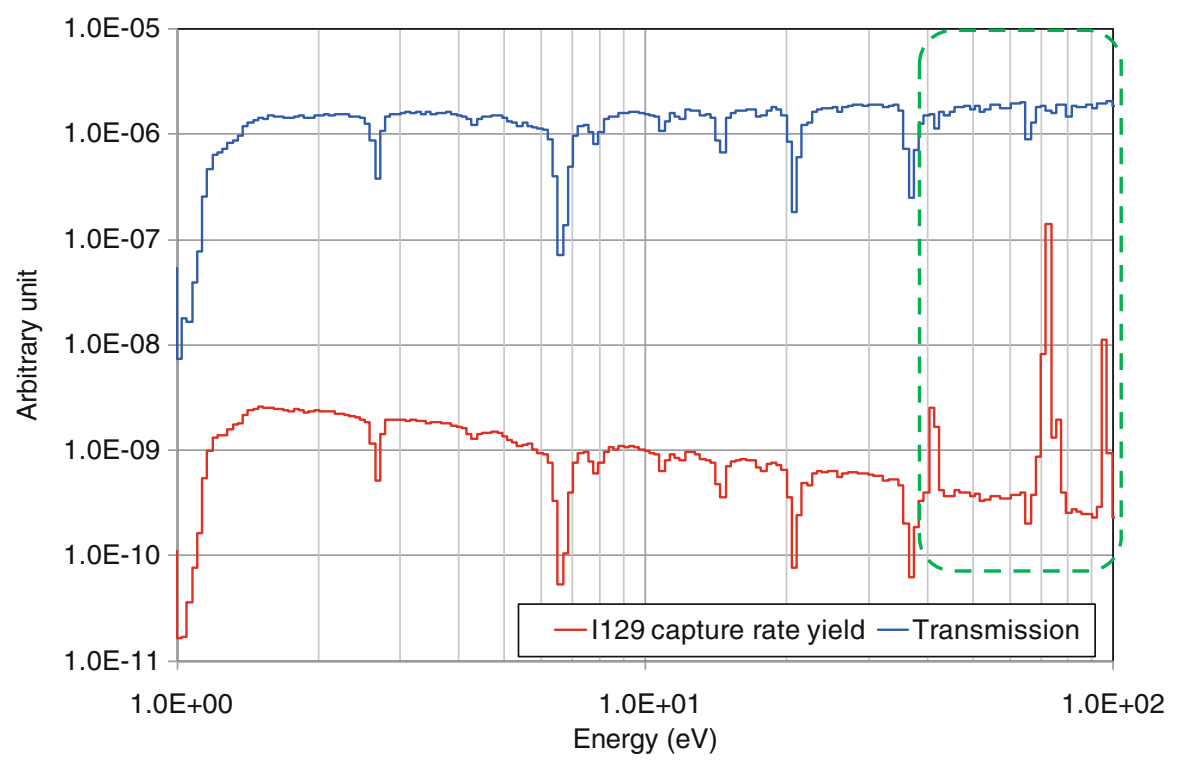

Fig. 4.4 I-129 absorption yield in an indicator (sample, $20 \mathrm{GWd} / \mathrm{t}$ )

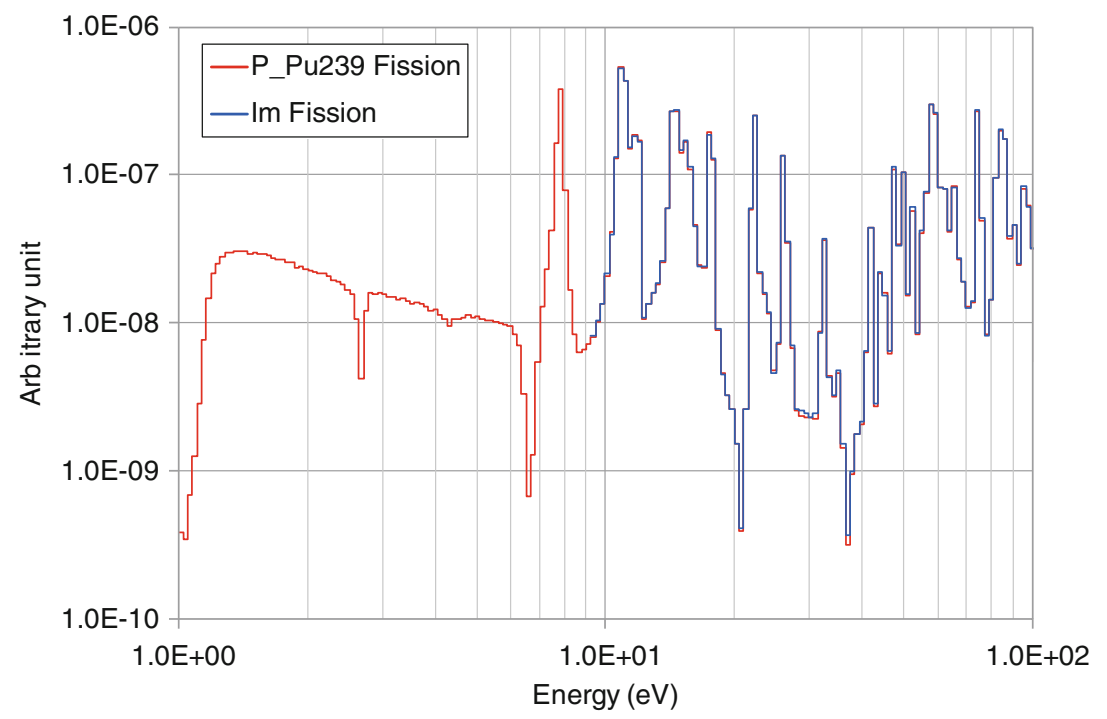

Fig. 4.5 Pu-239 absorption yield in an impure indicator (sample, $0 \mathrm{GWd} / \mathrm{t}$ )

Next, the applicability of the present method for fuel debris in Fukushima Daiichi NPP is examined. The fuel debris in Fukushima Daiichi NPP contains highly concentrated B-10, which has a large neutron absorption cross section. Thus, numerical validation of the present method and the conventional neutron transmission method for the sample with B-10 were carried out. The burn-up of the 


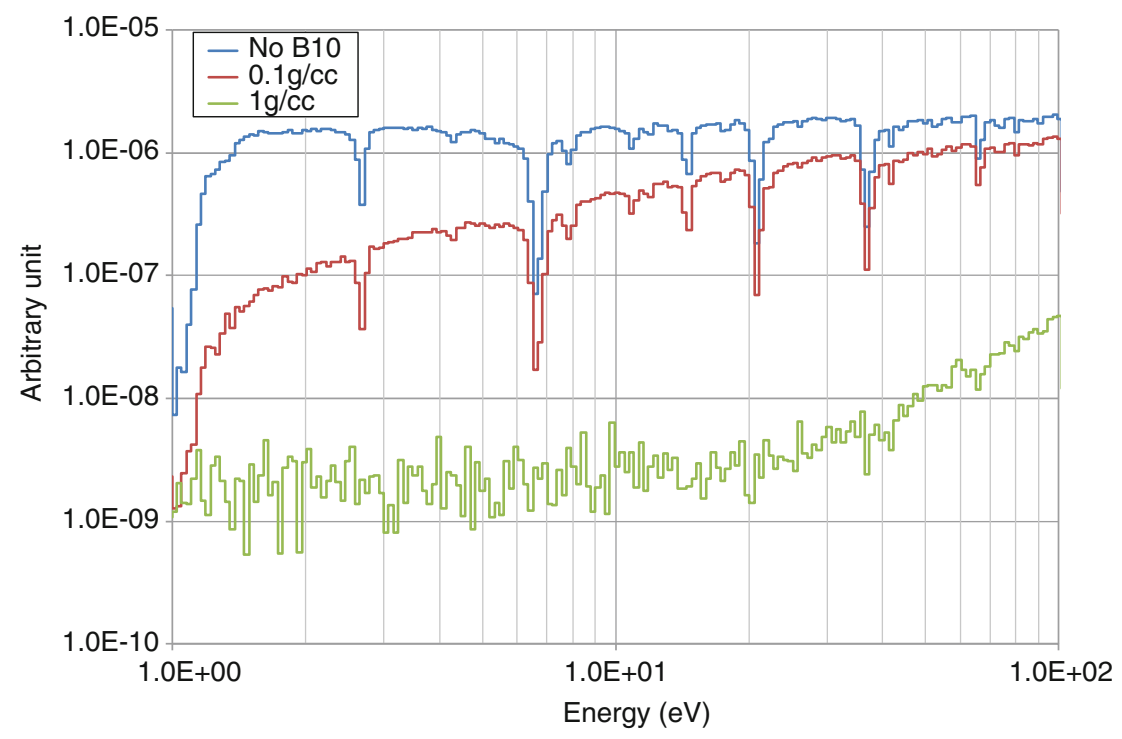

Fig. 4.6 Transmitted neutron spectrum from the sample $(30 \mathrm{GWd} / \mathrm{t})$ with B-10 (conventional method)

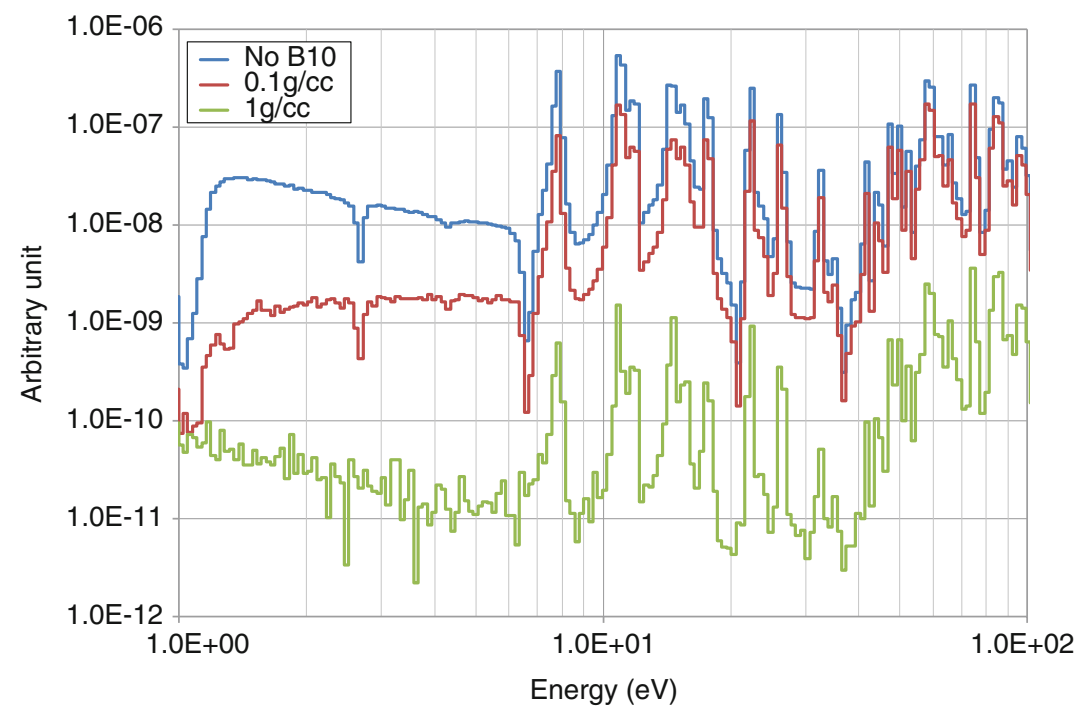

Fig. 4.7 Pu-239 absorption yield by self-indication method (sample, $30 \mathrm{GWd} / \mathrm{t}$ )

sample is $30 \mathrm{GWd} / \mathrm{t}$. Using the transmission neutron method, it is difficult to obtain the dips caused by resonance reaction (Fig. 4.6) because neutron absorption by B-10 has a large contribution in the sample. On the other hand, one can obtain the neutron absorption rate yield in an indicator by the present method although the signal of the neutron is decreased (Fig. 4.7). 


\subsection{Conclusion}

Numerical validation for application of the self-indication method has been carried out. As a result, the self-indication method is shown to have a better $\mathrm{S} / \mathrm{N}$ than the neutron transmission method to quantify the amount of target nuclides.

The present method can be applied to identify and quantify a nuclide that has a small resonance, i.e., ${ }^{129} \mathrm{I}$, and it is shown that one can measure an intended signal with good $\mathrm{S} / \mathrm{N}$ by using an impure indicator. In addition, if the sample contains a highly concentrated neutron absorber, one can identify and quantify the target nuclide by using the self-indication method. Thus, the self-indication method can be applied to analyze the fuel debris in Fukushima Daiichi NPP.

Acknowledgments This work was supported by JSPS KAKENHI Grant Number 24760714.

Open Access This chapter is distributed under the terms of the Creative Commons Attribution Noncommercial License, which permits any noncommercial use, distribution, and reproduction in any medium, provided the original author(s) and source are credited.

\section{References}

1. Working Party on Physics of plutonium fuels and innovative fuel cycles (2003) Physics of Plutonium fuels BWR MOX benchmark specification and results. OECD/NEA, ISBN: 92-6419905-5

2. Okumura K et al (2007) SRAC 2006: a comprehensive neutronics calculation code system. JAEA-Data/Code, 2007-004

3. Shibata K et al (2011) JENDL-4.0: a new library for nuclear science and engineering. J Nucl Sci Technol 48(1):1-30

4. Nagaya $\mathrm{Y}$ et al (2005) MVP/GMVP II: general purpose Monte Carlo codes for neutron and photon transport calculations based on continuous energy and multigroup methods. JAERI 1348, Japan Atomic Energy Research Institute 